\title{
Performance comparison of LEACH, SEP and Z-SEP Protocols in WSN
}

\author{
Hamdy H. El-Sayed \\ Mathematics Department, Faculty of Science \\ Sohag University, Egypt
}

\begin{abstract}
Wireless sensor networks (WSN) have major scope to monitor, and analyze the phenomena in the real world in details as much as possible, in places to a large degree, very dusky, very high, or very dangerous for researchers to go. Simulation could be great helpful to detect that the shortest possible time to minimize the cost of WSN when design. Simulation provides controlled environmental status for development and optimizing the design parameters and the configuration alternatives. It also represents a good prudence into the effects of the different parameters and so helps to identify those parameters to obtain the great importance for system operation and design. Some nodes transmit data directly to base station in Zonal Stable Election Protocol (ZSEP) protocol whilst some clustering technique that used are send data to base station as well as in Stable Election Protocol (SEP) protocol. The Zonal Stable Election Protocol is implemented and compared with traditional Low Energy adaptive clustering hierarchy (LEACH) and Stable Election Protocol. Results of simulation showed that Zonal Stable Election Protocol is enhanced the stability period and end-toend delay and less overhead than Low Energy adaptive clustering hierarchy and Stable Election Protocol. This article also tests the node density effects on dead nodes, alive nodes and packet to base station.
\end{abstract}

\section{Keywords}

wireless sensor network, number of nodes, LEACH, SEP and Z-SEP protocols, Packet-to-BS, Dead nodes, alive nodes, Endto-End Delay, Overhead.

\section{INTRODUCTION}

Huge number of sensor nodes is being the Wireless Sensor Networks (WSNs) which it deployed randomly to monitor or environmental conditions, like, vibration, pressure, temperature, sound, motion or pollutants which it is in different locations. Development of WSN make evaluation of wireless communications, electronics and technological evolution because of their low cost and variety of applications such like as health, home and military e.g. Component of sensor nodes which it consists of make it capable sensing data, processing data and also communication for transmit or receive data. Protocols of these networks must be accurate and efficient working and must be self-organizing to make network work.

The most efficient routing protocols are the hierarchical routing protocols. Several protocols are classified as homogeneous networks. LEACH [1] is one of the first clustered based routing protocols for homogeneous network. LEACH protocol allocates same probability to all the nodes to become cluster head. In heterogeneous environment LEACH does not perform well. To improve efficiency of WSNs, SEP [2] was proposed. SEP is a two level heterogeneous protocol. SEP specify different probability for nodes on the foundation of their energy level. The Z-SEP protocol was proposed in [3],
Z-SEP enhanced the stability period and throughput than existing protocols like LEACH and SEP. However, SEP has not extra energy for higher level nodes efficiently.

In this paper we have compared a hybrid approach for transmitting data to base station. We used node density affected for many parameters like dead nodes, alive nodes and packet to BS also we test node density affected on overhead and end to end delay parameters. Our compared algorithms results that the Z-SEP protocol is enhanced the stability period other than LEACH and SEP, also network lifetime and throughput of the network are stable.

Section 2 shows the overview work. Section 3 describes the experimental and results. In section 4, the main conclusions are discussed. And in the last section, references are presented.

\section{2- OVERVIEW}

The most famous method to manage the energy in WSNs is clustering techniques. Low Energy Adaptive Clustering Hierarchy (LEACH), is a clustering protocol that has been used cluster-heads to manage and distribute the energy load between sensors of network [4]. However, LEACH is homogenous protocol. It is distributed algorithm each node is capable to select itself as a cluster head by choosing random number. For real life conditions it is very difficult for sensors to maintain their energy uniformly, so, introducing energy needy. Stable Election Protocol (SEP) [5], is a heterogeneous protocol. It is based on weighted election probabilities for each node which become a cluster head according to their respective energy. Enhanced Stable Election Protocol (E-SEP) [6] was proposed for three level hierarchies. ESEP presented an intermediate node which energy is between normal node and advance node. Nodes consider and elected themselves as cluster head of their energy level. The behavior of ESEP is same as in SEP. Zonal Stable Election Protocol (Z-SEP) [3][7] also for heterogeneous environment there is two level heterogeneity. The field is divided in to three zones: Zone 0 , Head Zone 1 and Head Zone 2. Normal nodes behavior is only deployed in zone 0 to reduce the energy and they transmit data directly to base station. Some of advanced nodes accordingly half of them are deployed in Head zone 1 and the other half in Head zone 2 and they use clustering technique to transmit data to base station. The results that has been obtained shows the stability period and throughput have been increased compared with LEACH and SEP. two types of nodes and two level hierarchies were considered. SEP prolong the time interval before the death of the first node, which is crucial for many applications where the feedback from the sensor network must be reliable[8][9].

This article work test small network density nodes in the LEACH, SEP and Z-SEP protocols as the first part of simulation. The second part uses large network density node 
effects on number of dead nodes, alive nodes and packet to base station as a parameters for test.

\section{3- EXPERIMENTAL AND RESULTS}

The LEACH, SEP and Z-SEP protocols have been simulated using matlab 2015a and the parameters are listed in the table 1. Also we are used small network with little number of nodes and large network with large number of network nodes to test the performance of these protocols. The parameters that have been used to test the comparison are Packet-to-BS, Dead nodes and Alive nodes in the network.

\section{Table 1: list of simulation parameters}

\begin{tabular}{|c|c|c|}
\hline $\begin{array}{l}\text { S. } \\
\text { No. }\end{array}$ & Parameters & Values \\
\hline 1 & Network Area & $300 * 300$ \\
\hline 2 & Number of Nodes & $10,20,1000,2000$ \\
\hline 3 & $\begin{array}{ll}\text { Cluster } & \text { head } \\
\text { probability } & \end{array}$ & 0.3 \\
\hline 4 & Base station location & $(\mathbf{5 0 , 5 0 )}$ \\
\hline 5 & transmiter energy & $50 * 0.000000001$ \\
\hline 6 & reciever energy & $50 * 0.000000001$ \\
\hline 7 & Aggregation Energy & $50 * 0.000000001$ \\
\hline 8 & amplification energy & $0.0013 * 0.000000000001$ \\
\hline 9 & Number of Rounds & 9000 \\
\hline 10 & Hard Threshold & 100 \\
\hline 11 & Soft Threshold & 2 \\
\hline
\end{tabular}

\section{1- First Simulation}

A clustered wireless sensor network has been simulated in a field with dimensions $300 \mathrm{~m} \times 300 \mathrm{~m}$. The population of the sensors nodes in the network is equal $10(\mathrm{n}=10)$. The nodes distribution is random over the field. We placed the BS at a far distance from all other nodes. The results for $50 \mathrm{~m} \mathrm{x}$ $50 \mathrm{~m}$ have ploted when our BS is located at $(50,50)$. So that the BS is at least $50 \mathrm{~m}$ from the closest sensor node.

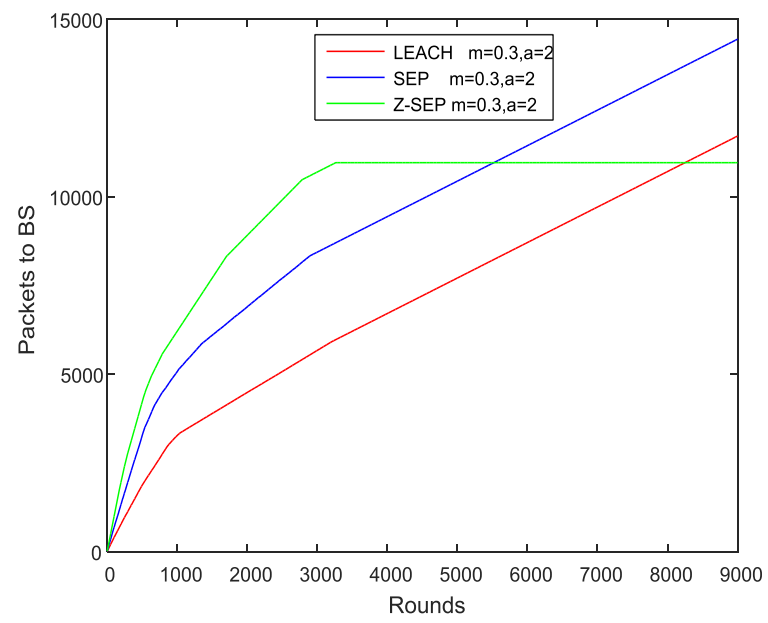

Figure 1: Packet-To-BS In Network With 10 Nodes

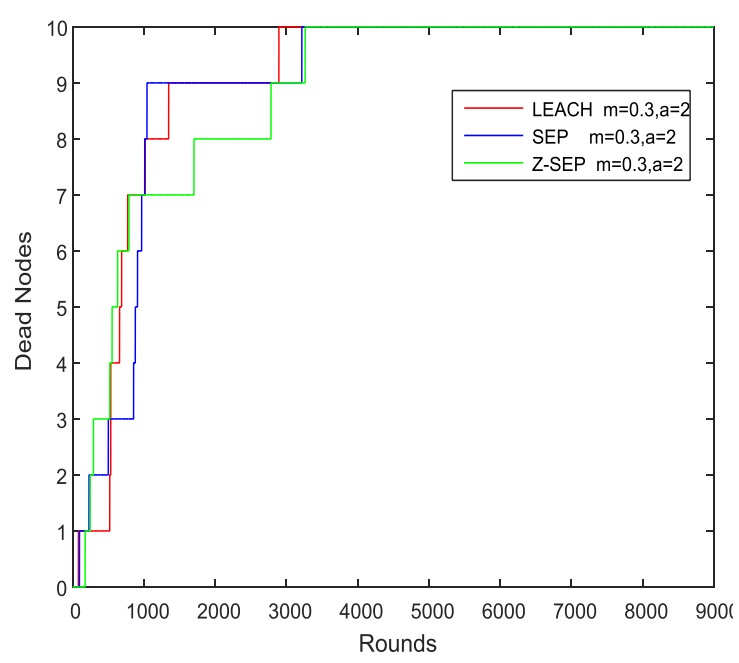

Figure 2: Dead Nodes In Network With 10 Nodes

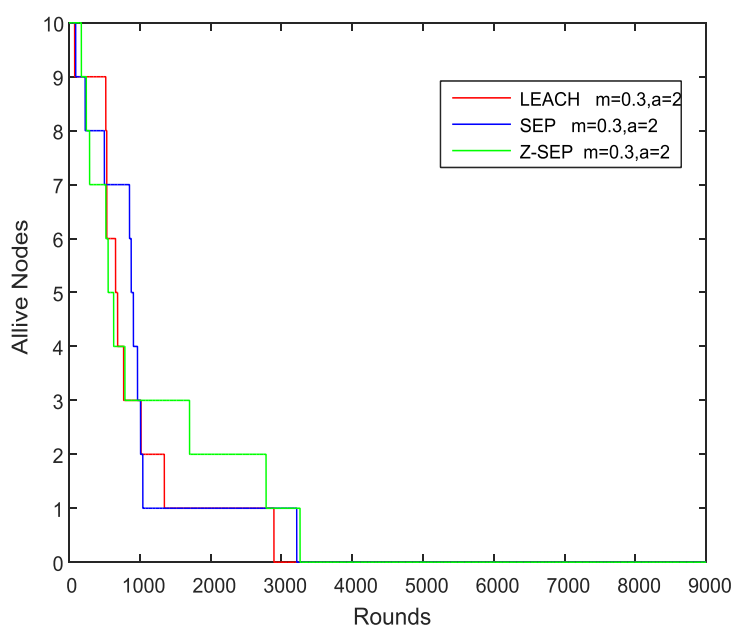

Figure 3: Alive Nodes In Network With 10 Nodes

From figure 1,2 and 3 we can see that the Z-SEP stability period is almost same for the cases $(\mathrm{m}=0.3$ and $\mathrm{a}=2)$ with 10 nodes number of sensor network than SEP and LEACH. The reason is that the normal nodes have the same amount of energy, they have the same amount of energy and they die almost at the same time, however network lifetime is increased because of the extra energy of advance nodes. LEACH stability period is decreased because the LEACH protocol is more sensitive to heterogeneity. LEACH is did not have weighted probability like in SEP for even distribution of more extra energy. In LEACH every node has equal chance to become cluster head so normal nodes die sooner than advance nodes [7][8].

\section{2- Second Simulation}

Secondly a clustered wireless sensor network has been simulated in a field with dimensions $300 \mathrm{~m} \times 300 \mathrm{~m}$. The population of the sensors nodes in the network is equal 20 $(\mathrm{n}=20)$. The nodes distribution is randomly over the field. We placed the BS at a far distance from all other nodes. The results for $50 \mathrm{~m} \times 50 \mathrm{~m}$ are plot when our BS is located at $(50,50)$. So that the BS is at least $50 \mathrm{~m}$ from the closest sensor node. 


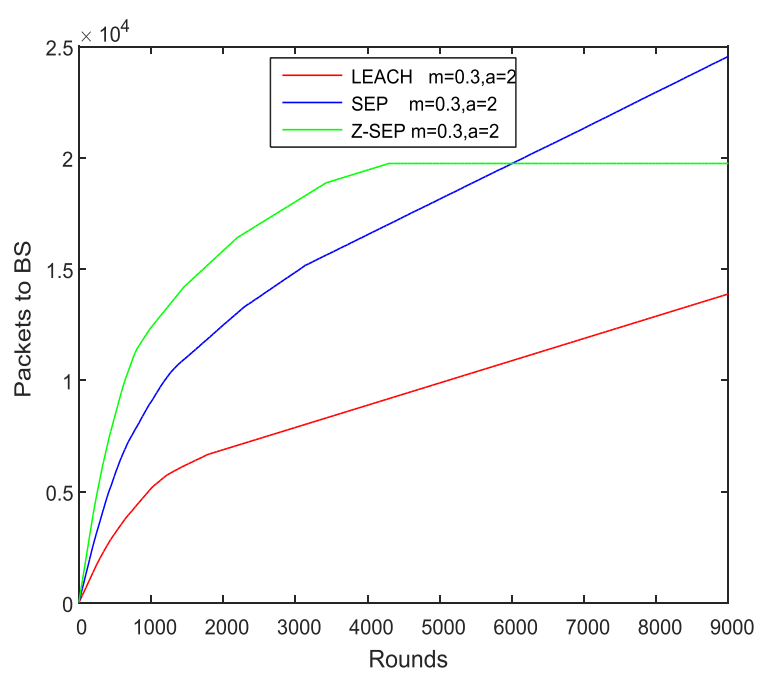

Figure 4: Packet-To-BS In Network With 20 Nodes

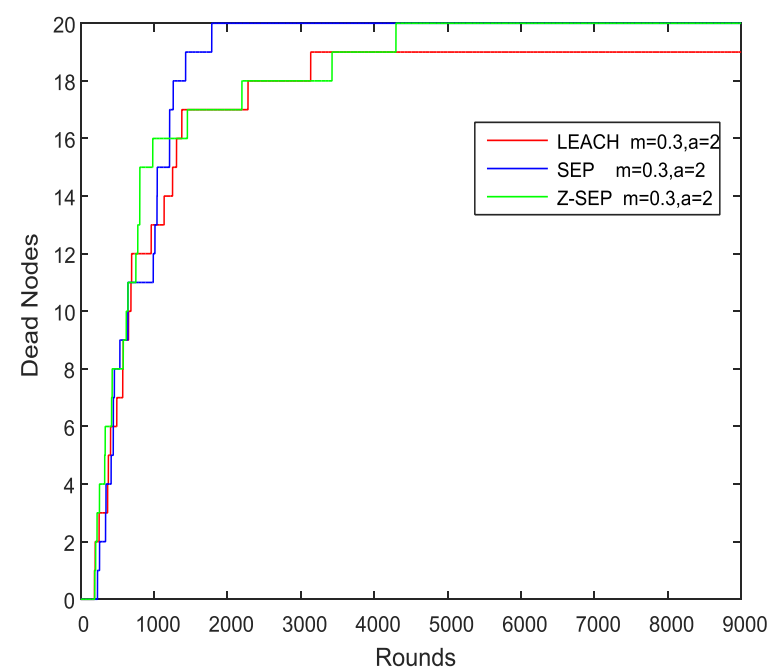

Figure 5: Dead Nodes In Network With 20 Nodes

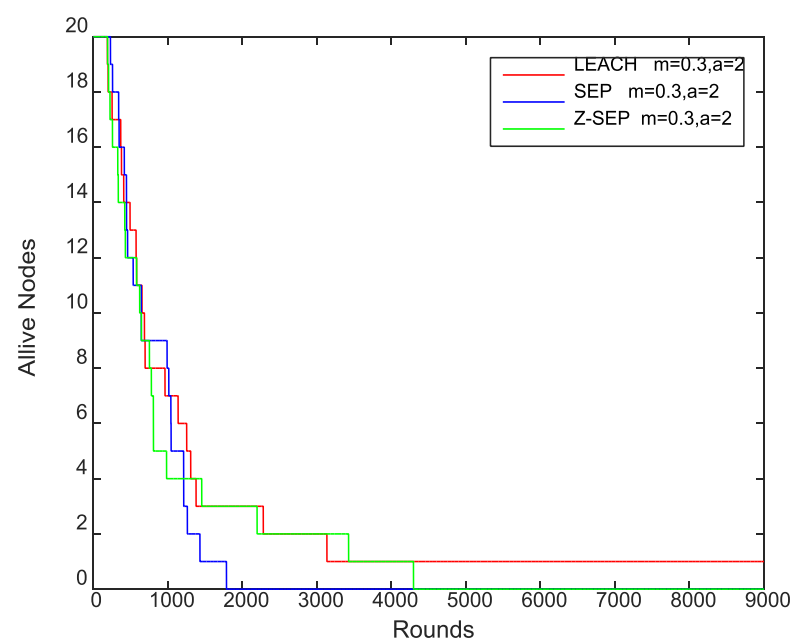

Figure 6: Alive Nodes In Network With 20 Nodes

Figure 4 shows that the packet arrives to base station in Z-SEP more than SEP and LEACH but when round become 3000 and more packets to base station be stable and stop increasing.
Also the packet arrives to base station of SEP increases more than LEACH.

Figure 5 depicts that the dead nodes rate of SEP is more than Z-SEP and LEACH and be little stable by increasing number of rounds. Figure 6 shows that the SEP alive node is little less than others but the Z-SEP is more enhanced than LEACH and SEP protocols.

\section{3- Third Simulation}

A gain a clustered wireless sensor network has been simulated in a field with dimensions $300 \mathrm{~m} \times 300 \mathrm{~m}$. The population of the sensors nodes in the large network is equal to $1000(\mathrm{n}=1000)$. The nodes are randomly distributed over the field. The BS is placed at a far distance from all other nodes. The results $50 \mathrm{~m} \times 50 \mathrm{~m}$ are plots when our BS is located at $(50,50)$. So that the BS is at least $50 \mathrm{~m}$ from the closest sensor node.

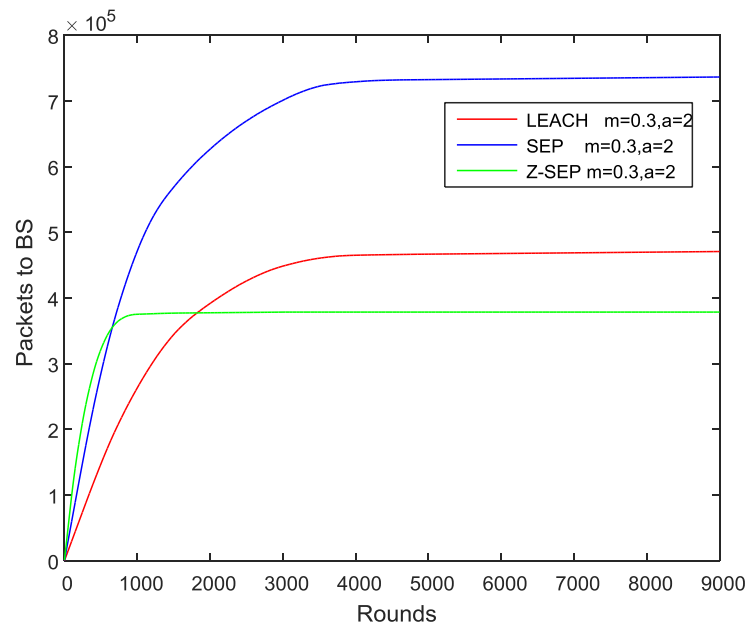

Figure 7: Packet-To-BS In Network With 1000 Nodes

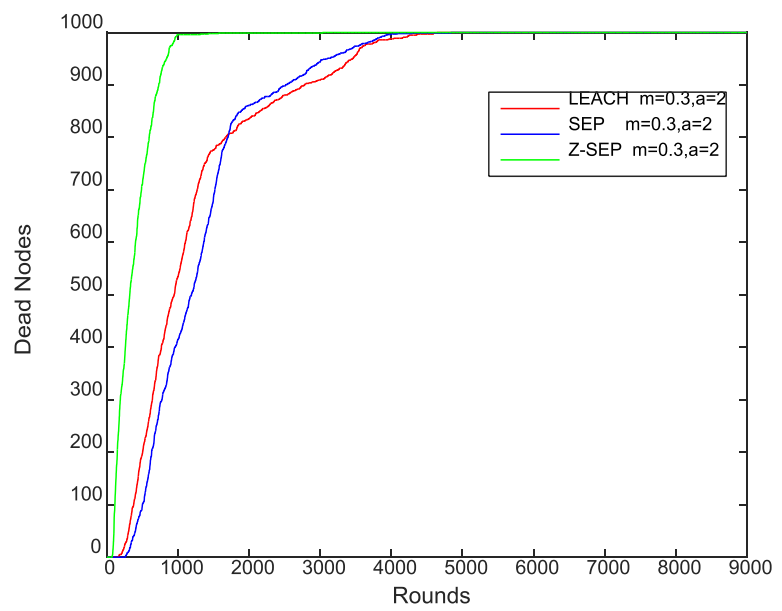

Figure 8: Dead Nodes In Network With 1000 Nodes 


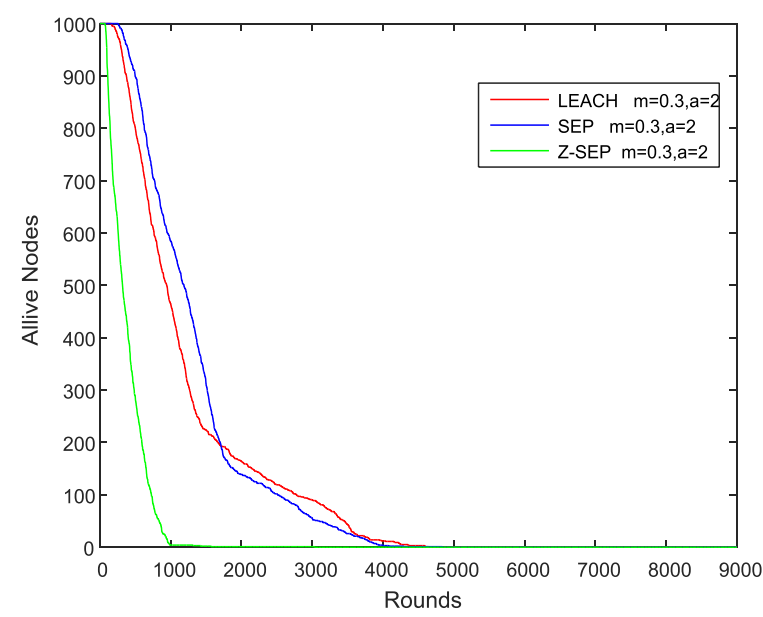

Figure 9: Alive Nodes In Network With 1000 Nodes

Figure 7,8,9 shows that the packet arrives to base station in ZSEP more than SEP and LEACH but when round become 1000 and when rounds be more the packets to base station be stable and stop to increasing. Also the packet arrives to base station of SEP increases more than LEACH and LEACH more than Z-SEP. Z-SEP performs better than LEACH and SEP, because nodes in the head zone 0 which it normal nodes communicates directly to the base station otherwise nodes in head zone 1 and head zone 2 communicates via cluster head to base station: As in clustering technique, cluster head consumes energy in the form of data aggregation and also by receiving data from nodes in the cluster. LEACH stability period is decreased because the LEACH protocol is more sensitive to heterogeneity. LEACH is did not have weighted probability like in SEP for even distribution of more extra energy. Z-SEP is more consolidated than LEACH and SEP protocols.

\section{4- Fourth Simulation}

Lastly a clustered wireless sensor network has been simulated in a field with dimensions $300 \mathrm{~m} \times 300 \mathrm{~m}$. The population of the sensors nodes in the large network is equal to 2000 ( $n=$ 2000). The nodes are randomly distributed over the field. We placed the BS at a far distance from all other nodes. We simulate the results for $50 \mathrm{~m} \times 50 \mathrm{~m}$ plot when our BS is located at $(50,50)$ so that the BS is at least $50 \mathrm{~m}$ from the closest sensor node.

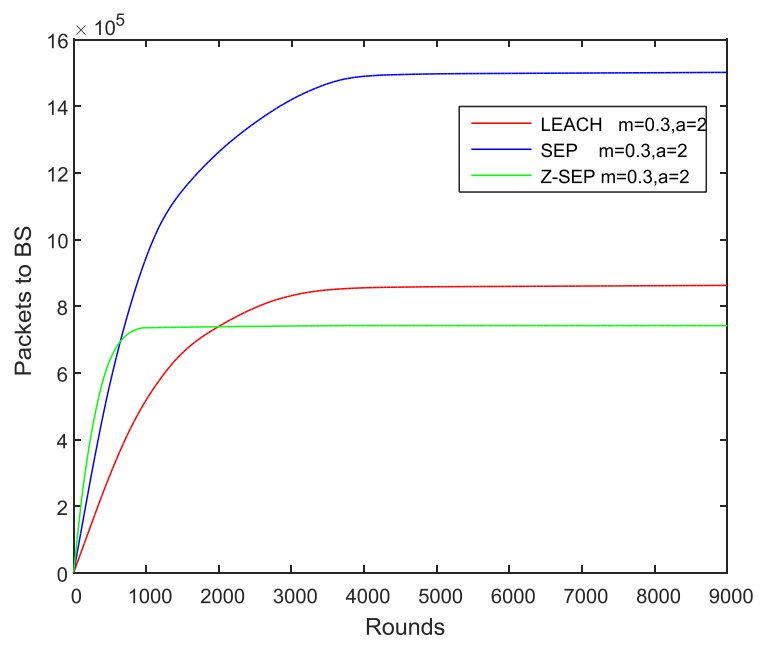

Figure 10: Packet-To-BS In Network With 2000 Nodes

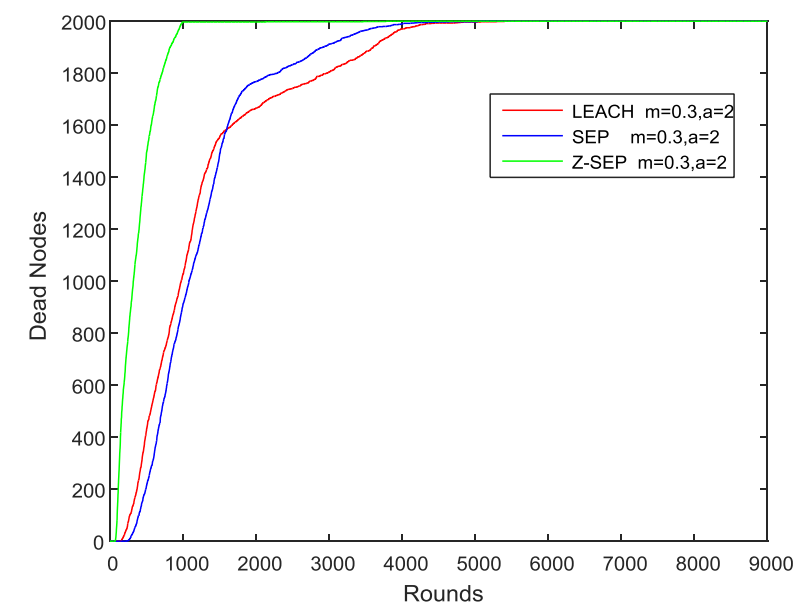

Figure 11: Dead Nodes In Network With 2000 Nodes

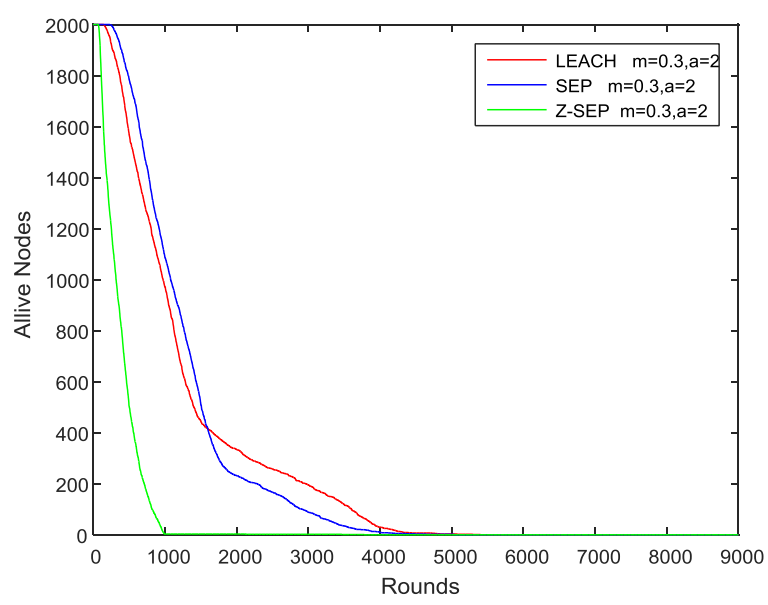

Figure 12: Alive Nodes In Network With 2000 Nodes

From figure 10, we see that the packet arrives to base station in Z-SEP more than SEP and LEACH but when round become little less than 1000 and rounds be more the packets to base station be stable and stop to increasing. Also the packet arrives to base station of SEP increases more than LEACH and LEACH more than Z-SEP. also from figure 11 and 12 we can see that the Z-SEP stability period is almost same for the cases $(\mathrm{m}=0.3$ and $\mathrm{a}=2)$ with 2000 nodes number of sensor large network. Z-SEP performs better than LEACH and SEP, because nodes in the head zone 0 which it normal nodes communicates directly to the base station otherwise nodes in head zone 1 and head zone 2 communicates via cluster head to base station: As in clustering technique, cluster head consumes energy in the form of data aggregation and also by receiving data from nodes in the cluster. LEACH stability period is decreased because the LEACH protocol is more sensitive to heterogeneity. The stability period of Z-SEP protocol is increased from LEACH and SEP, however network lifetime is decreased when compared with LEACH. From compared the SEP and Z-SEP protocols the network life time is increased due to advance nodes which die slower than normal nodes.

Both of each LEACH, SEP and Z-SEP protocols the overhead increases with increased number of nodes or size of network. We could define the overhead as follows "routing and data packets have to share the same network bandwidth most of times and hence routing packets are considered to be overhead 
in the network". So a good routing protocol which has a less overhead. From figure 13 and table 2 the Z-SEP protocol has less overhead the LEACH and SEP protocols. the packet that are sends by the Z-SEP protocol takes less time than other protocols like LEACH and SEP.

Table 2: Overhead Protocols tests on LEACH, SEP and ZSEP

\begin{tabular}{|l|l|l|l|}
\hline \multirow{2}{*}{$\begin{array}{l}\text { N. of } \\
\text { nodes }\end{array}$} & LEACH & SEP & Z-SEP \\
\cline { 2 - 4 } & Overhead & Overhead & Overhead \\
\hline \hline 10 & $8.5903 \mathrm{e}-04$ & $7.4828 \mathrm{e}-04$ & $5.5769 \mathrm{e}-04$ \\
\hline 20 & 0.0014 & 0.0014 & $8.8017 \mathrm{e}-04$ \\
\hline 100 & 0.0022 & 0.0021 & 0.0012 \\
\hline 200 & 0.0022 & 0.0025 & 0.0013 \\
\hline 1000 & 0.0024 & 0.0027 & 0.0014 \\
\hline 2000 & 0.0024 & 0.0027 & 0.0014 \\
\hline
\end{tabular}

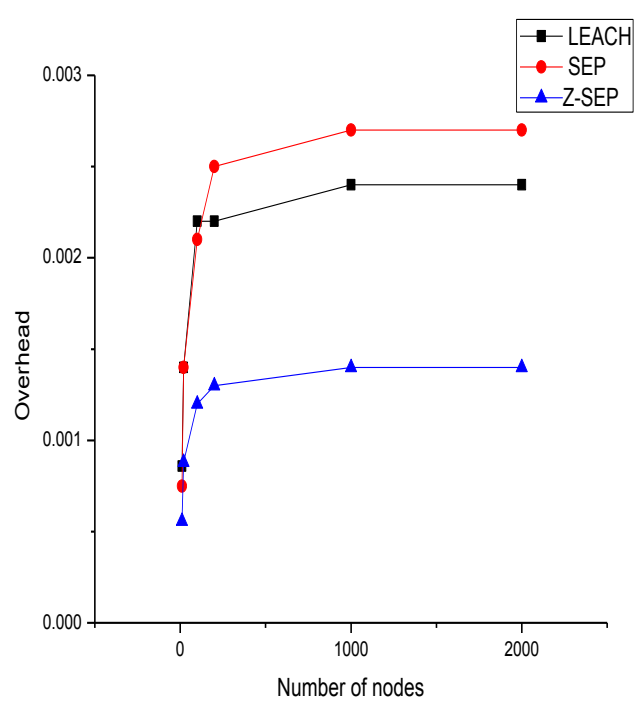

Figure 13: Overhead Protocols tests on LEACH, SEP and Z-SEP with different number of nodes

Both of each LEACH, SEP and Z-SEP protocols the delay increases with increased number of nodes in the network which end-to-end delay means the time taken by a packet to travel from source to destination depends on the number of hops and congestion on the network. By increasing number of node the hops and congestion increased which delay increase means that the packet take times a lot to arrive to destination this is showed in figure 14 and table 3 for the LEACH, SEP and Z-SEP protocols. But the Z-SEP protocol is faster than others.

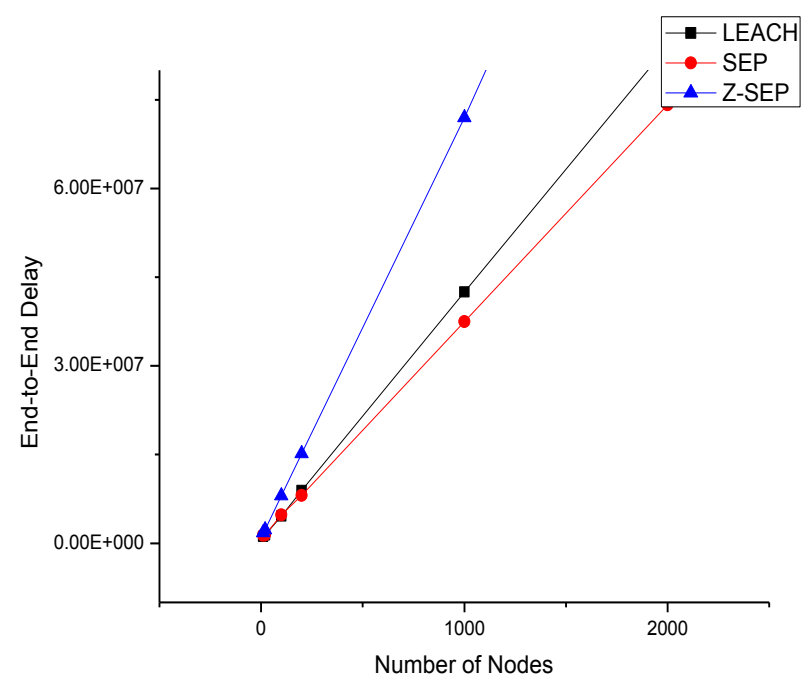

Figure 14: End-to-End Delay Protocols tests on LEACH, SEP and Z-SEP with different number of nodes

Table 3: End-to-End Delay tests on LEACH, SEP and Z-SEP

\begin{tabular}{|l|l|l|l|}
\hline \multirow{2}{*}{$\begin{array}{l}\text { N. of } \\
\text { nodes }\end{array}$} & LEACH & SEP & Z-SEP \\
\cline { 2 - 4 } & $\begin{array}{l}\text { End-to-End } \\
\text { Delay }\end{array}$ & $\begin{array}{l}\text { End-to-End } \\
\text { Delay }\end{array}$ & $\begin{array}{l}\text { End-to-End } \\
\text { Delay }\end{array}$ \\
\hline \hline 10 & 1164100 & 1336400 & 1793100 \\
\hline 20 & 1401500 & 1387300 & 2272300 \\
\hline 100 & 4593100 & 4789600 & 8037100 \\
\hline 200 & 8912900 & 8065300 & 15156300 \\
\hline 1000 & 42518100 & 37463900 & 71974900 \\
\hline 2000 & 84052700 & 74146300 & 147987300 \\
\hline
\end{tabular}

\section{4- CONCLUSION}

In this paper, the LEACH, SEP and Z-SEP are compared for heterogeneous environment. The comparison was in small with 10 and 20 nodes and in the other side large network with 1000 and 2000 nodes. Results have shown that the stability period of Z-SEP is increased but in LEACH decreased. by just altering the deployment of the different type of nodes in different zones according to their energy requirement. the SEP and Z-SEP protocols the network life time is increased due to advance nodes which die slower than normal nodes. Our novelty of this work is to test the small number of nodes and large number of node effects on the performance of LEACH, SEP and Z-SEP protocols using Packet-to-BS, Dead nodes and alive nodes as a parameters also we test the overhead and end-to-end delay in both small and large network. Z-SEP protocol has less overhead the LEACH and SEP protocols. the packet that are sends by the Z-SEP protocol takes less time than other protocols like LEACH and SEP. Both of each LEACH, SEP and Z-SEP protocols the delay increases with increased number of nodes in the network. Packet to base station is decreased by increasing number of nodes for the three protocols but in Z-SEP protocol it is decreased a lot than others. It reach to stable stage quickly. 


\section{REFERENCES}

[1] Heinzelman, W. R., Chandrakasan, A., \& Balakrishnan, H:"Energy-efficient communication protocol for wireless microsensor networks", In System Sciences, Proceedings of the 33rd Annual Hawaii International Conference on (pp. 10-pp). IEEE, January, 2000.

[2] Smaragdakis, G., Matta, I., \& Bestavros, A. SEP: A stable election protocol for clustered heterogeneous wireless sensor networks. Boston University Computer Science Department, 2004.

[3] S. Faisal , N. Javaid, A. Javaid, M. A. Khan, S. H. Bouk and Z. A. Khan "Z-SEP: Zonal-Stable Election Protocol for Wireless Sensor Networks", Journal of Basic and Applied Scientific Research (JBASR), 2013.

[4] [4] Heinzelman, A. P. Chandrakasan, and $H$. Balakrishnan "An application-specific protocol architecture for wireless microsensor networks," IEEE Transactions on Wireless Communications, vol. 1, no. 4, pp. 660-670, October 2002.

[5] [5] G. Smaragdakis, I. Matta, A. Bestavros, "SEP: A Stable Election Protocol for clustered heterogeneous wireless sensor networks", in: Second International
Workshop on Sensor and Actor Network Protocols and Applications (SANPA 2004), 2004.

[6] [6] Aderohunmu, F. A., \& Deng, J. D. An Enhanced Stable Election Protocol (SEP) for Clustered Heterogeneous WSN (No. 2009/07), Discussion Paper Series, 2009.

[7] [7] Banoth Thirumala, B. Vijay Kumar and Aloth Sowjanya: "Zonal-Stable Election Protocol for Wireless Sensor", International Journal of Ethics in Engineering \& Management Education, Volume 3, Issue 10, October 2016.

[8] [8] Upasana Sharma, Sunil Tiwari, "Performance Analysis of SEP and LEACH for Heterogeneous Wireless Sensor Networks", International Journal of Computer Trends and Technology (IJCTT) - volume 10 number 4-Apr 2014.

[9] [9] Hamdy H. El-Sayed :" Data Aggregation Energy and Probability Effects on the Performance of EDEEC and MODLEACH Protocol in WSN", Appl. Math. Inf. Sci. 12, No. 1, 1-7 (2018). 